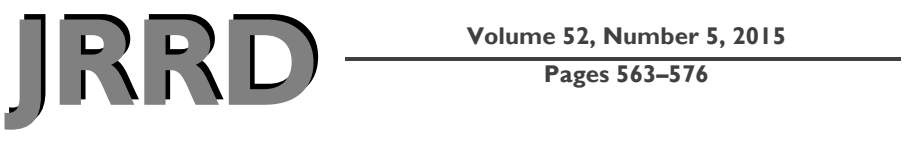

\title{
Impact of comorbid depression on quality of life in male combat Veterans with posttraumatic stress disorder
}

\author{
Phillip A. Raab, MA; ${ }^{\text {* }}$ Margaret-Anne Mackintosh, PhD; ${ }^{1}$ Daniel F. Gros, PhD; ${ }^{2}$ Leslie A. Morland, PsyD $^{1,3}$ \\ ${ }^{1}$ National Center for Posttraumatic Stress Disorder, Pacific Islands Division, Department of Veterans Affairs (VA) Pacific \\ Islands Health Care System, Honolulu, HI; ${ }^{2}$ Ralph H. Johnson VA Medical Center, Charleston, SC; and Medical Univer- \\ sity of South Carolina, Charleston, SC; ${ }^{3}$ John A. Burns School of Medicine, University of Hawaii at Manoa, Honolulu, HI
}

\begin{abstract}
For Veterans with posttraumatic stress disorder (PTSD), depression is a highly comorbid condition. Both conditions have been associated with decreased quality of life, and research suggests that comorbid PTSD and depression may result in worse quality of life than PTSD alone. However, research is needed to elucidate the effect of comorbidity on a broader variety of quality of life domains. In this study, we used baseline data of 158 male combat Veterans taking part in a PTSD treatment trial and examined the unique relationships between quality of life domains and PTSD symptom clusters, major depressive disorder (MDD) diagnosis, and self-reported depressive symptoms. Veterans with comorbid PTSD-MDD reported significantly worse satisfaction-related quality of life than those with PTSD alone, although this finding was largely attributable to PTSD numbing symptoms. Subsequent analyses comparing the effect of numbing symptoms to depressive symptoms revealed that depression exerted a stronger influence, although numbing symptoms were still uniquely associated with quality of life. We discuss implications for treatment and research, as well as the need to address negative affect in Veterans with PTSD.
\end{abstract}

Clinical Trial Registration: ClinicalTrials.gov; NCT00879255; "Telemental Health and Cognitive Processing Therapy for Rural Combat Veterans with Posttraumatic Stress Disorder (CPTVTEL)" (parent study from which present study draws its data); http://www.clinicaltrials.gov/ct2/show/NCT00879255

Key words: comorbidity, depression, major depressive disorder, MDD, negative affect, numbing, posttraumatic stress disorder, PTSD, quality of life, Veterans.

\section{INTRODUCTION}

Research has demonstrated that for Veterans with posttraumatic stress disorder (PTSD), depression is a highly comorbid condition [1]. A recent large meta-analysis composed of 57 studies, across both military and civilian samples, reported a comorbidity rate of 52 percent [2]. The underlying reason for this high degree of comorbidity is unclear. One theory postulates that the disorders represent differing symptom manifestations of an underlying stress

Abbreviations: BDI-II = Beck Depression Inventory-II, CAPS = Clinician-Administered PTSD Scale for DSM-IV, CI = confidence interval, DSM = Diagnostic and Statistical Manual of Mental Disorders, MCS = SF-12 Mental Component Summary, $\mathrm{MDD}=$ major depressive disorder, $\mathrm{OEF}=$ Operation Enduring Freedom, OIF $=$ Operation Iraqi Freedom, PCL $=$ Posttraumatic Stress Disorder Checklist, PCL-M = PCL-Military Version, PCL-S = PCL-Stressor Specific Version, PCS = SF-12 Physical Component Summary, PTSD = posttraumatic stress disorder, QOLI $=$ Quality of Life Inventory, SCID $=$ Structured Clinical Interview for DSM-IV Axis I Disorders, SF-12 $=12$-Item Short Form Health Survey, VA = Department of Veterans Affairs.

*Address all correspondence to Phillip A. Raab, MA; National Center for Posttraumatic Stress Disorder, Pacific Islands Division, 3375 Koapaka St, Suite I-560, Honolulu, HI 96819; 808-566-1546; fax: 808-566-1885.

Email: drewraab@gmail.com

http://dx.doi.org/10.1682/JRRD.2014.05.0130 
construct, a position supported by the common diatheses of the two disorders [3]. Researchers have also highlighted the lack of specificity of PTSD's symptoms as a possible cause, and a recent study with a Veteran sample found that a subset of PTSD symptoms related to numbing loaded more strongly on a depression factor than on the PTSD factor [4]. Furthermore, in that study, individuals endorsing these numbing-related symptoms reported significantly more comorbid PTSD and depression and significantly higher depressive symptom severity.

While there is no definitive explanation for the high degree of depression comorbidity observed in individuals with PTSD, there is reason to suspect that it may be associated with reduced Veteran quality of life, which has been conceptualized as a person's physical, mental, and social well-being [5]. Research has shown that PTSD has a deleterious impact on Veteran life quality, as Veterans with PTSD report increased rates of unemployment due to disability [6], decreased social [7] and occupationrelated functioning [8], and lower life satisfaction [9]. There is also literature documenting the significant negative impact of depression on quality of life. Similar to PTSD, depression has been associated with financial problems [10] and decreased life satisfaction [11].

Several studies suggest that individuals with PTSD who also have comorbid depression have worse quality of life than those with PTSD alone. Research in nonVeteran samples has found that PTSD and depression comorbidity is associated with significantly worse major role functioning than PTSD alone [12] and significantly worse social and occupational functioning than is associated with only PTSD or neither disorder [13]. The few studies conducted with Veterans have produced a similar pattern of findings. In a study with Israeli Veterans of the 1982 Lebanon War, individuals with comorbid PTSD, depression, and anxiety reported worse psychosocial functioning than individuals with only PTSD [14]. Additionally, in a large sample of Korean war Veterans, individuals with comorbid PTSD and depression reported poorer satisfaction with their physical and psychological health, social relationships, and environment compared with Veterans with only PTSD or only depression [15]. These studies provide preliminary evidence that PTSD and comorbid depression can have a significantly more detrimental impact on Veteran quality of life than PTSD alone. However, further research is needed to elucidate this impact, given the multifaceted nature of quality of life.
Quality of life has been operationalized in a variety of ways and using various measures, with researchers advocating the importance of assessing both subjective life satisfaction [16] as well as more objective indices [17]. In their review of the quality of life literature for Iraq and Afghanistan Veterans, Schnurr and colleagues [18] adopted a framework focusing on subjective and objective indices across three domains: social-material conditions, functioning, and satisfaction [17]. Social-material quality of life includes areas such as employment, income, and marital status. Functioning involves an individual's role performance, or their ability to function at work, in relationships, and in different life domains. Satisfaction is concerned with individual well-being and subjective appraisal of life quality [18]. This tripartite approach shares similarities to other conceptualizations of quality of life [19], as well as the World Health Organization's definition of the construct [5]. Existing studies have examined the impact of comorbid PTSD and depression on these domains [14-15], but more research is needed exploring these relationships in Veteran samples.

Additionally, further empirical investigation is needed to disentangle the unique contributions of PTSD and depression to the decreased quality of life associated with comorbidity of the disorders [18]. One study involving Operation Iraqi Freedom (OIF)/Operation Enduring Freedom (OEF) combat Veterans found that PTSD and depression both independently contributed to mental-health-related quality of life, with depression predicting more variance than PTSD [20]. In another study of Veterans, depressive symptoms had a significant impact on satisfaction-related quality of life, although PTSD was not significantly related [21]. Likewise, in a sample of Veterans with PTSD, researchers found that depression predicted overall physical-health-related quality of life, while PTSD did not, although analysis of the quality of life subscales provided a more nuanced picture of the findings. While depressive symptoms predicted physical and role functioning scales, both depressive symptoms and PTSD independently predicted general health and bodily pain scales, and depression and PTSD interacted to predict vitality and social functioning scales [22]. Taken together, the existent research suggests that PTSD and depression make unique contributions to decreased quality of life, but that depression exerts a stronger influence. Further research is needed, however, especially to examine possible differences in the impact of depression and individual PTSD symptom clusters. Studies have found that various PTSD symptom clusters are negatively associated with quality of 
life [7,23], perhaps most notably the PTSD numbing symptom cluster [9,23-24], identified in the emotional numbing four-factor structure [25] and later incorporated into the structure of the Diagnostic and Statistical Manual of Mental Disorders (DSM)-5 diagnostic criteria for PTSD [26]. Because the numbing cluster shares significant overlap with depression $[1,4]$, understanding its relationship with quality of life and how it resembles or differs from that of the other symptom clusters, is essential to understanding the unique contributions of PTSD and depression to decreased quality of life.

The purpose of the current study was to examine the impact of PTSD and comorbid depression on socialmaterial, functioning, and satisfaction-related quality of life domains in a sample of male combat Veterans with PTSD. We used the DSM-IV PTSD symptom clusters of the emotional numbing model [25], which share significant overlap with the new DSM-5 diagnostic criteria [26]. For all domains where comorbid depression was significantly associated with poorer quality of life, we explored the unique contributions of PTSD symptom clusters and depression. While we initially defined depression by categorical diagnosis of major depressive disorder (MDD), we also conducted post hoc analyses operationalizing depression using the Beck Depression Inventory-II (BDI-II) [27], which provided a dimensional measure of depressive symptoms.

We predicted that individuals with comorbid PTSD and MDD would have significantly worse quality of life than individuals with PTSD alone across all social-material, functioning, and satisfaction-related quality of life domains. Given the research connecting a variety of PTSD symptom clusters to poorer quality of life, we also predicted that both PTSD symptom clusters and comorbid MDD would uniquely influence each quality of life domain.

\section{METHODS}

\section{Participants}

We drew data for this study from the baseline assessments of a clinical trial evaluating the effectiveness of the telehealth modality for delivering cognitive processing therapy [27] to combat Veterans with PTSD [28]; this included baseline data from a pilot cohort [29]. Data were collected at Department of Veterans Affairs (VA) clinics, and all participants provided written informed consent prior to participation. Participants in the current study consisted of 158 male combat Veterans with PTSD, and the inclusion criterion for the current study was a diagnosis of current PTSD based on the Clinician-Administered PTSD Scale for DSM-IV (CAPS) [30]. Participants were primarily white $(n=70,45.2 \%)$, Pacific Islander $(n=31$, $20.0 \%)$, or Asian $(n=23,14.8 \%)$. They mainly served in the Vietnam $(n=100,63.3 \%)$, OIF/OEF $(n=47,29.7 \%)$, or Operations Desert Storm/Shield $(n=24,15.2 \%)$ theaters, and in the Army $(n=109,69.0 \%)$, Marines $(n=22$, $13.9 \%)$, Navy $(n=21,13.3 \%)$, and Air Force $(n=14$, $8.9 \%$ ). Because some Veterans served in multiple war eras or in multiple military branches, numbers and percentages are larger than the total sample size.

\section{Measures}

Participants completed psychometrically sound selfreport measures and clinician-administered interviews to assess PTSD and depression symptoms as well as quality of life domains. For clinician-administered interviews, master's- and doctoral-level assessors were provided initial training in interview administration and required to demonstrate sufficient fidelity before conducting independent assessments. All assessors were also provided ongoing supervision to maintain fidelity to assessment protocols, and a random 20 percent of CAPS protocols in the parent study were independently reviewed.

\section{Posttraumatic Stress Disorder}

\section{Clinician-Administered PTSD Scale for DSM-IV.}

The CAPS is a clinician-administered interview for diagnosis of DSM-IV PTSD [31]. The CAPS was used in this study to establish diagnosis of PTSD with the " $1 / 2$ " rule, in which symptoms must have occurred minimally within the last month and be of at least moderate intensity [30]. The CAPS has good convergent validity and strong reliability [31].

Posttraumatic Stress Disorder Checklist. The Posttraumatic Stress Disorder Checklist (PCL) is a 17-item selfreport questionnaire that uses a 5-point Likert scale to measure severity of the 17 DSM-IV PTSD symptoms [32]. The PCL-Military Version (PCL-M) was used with the first two cohorts of the parent study, and the PCL-Stressor Specific Version (PCL-S) was used in all other cohorts. The scales have nearly identical items but differ in their instructions, and research with a military sample suggests that changes to PCL-S instructions do not affect reporting of symptoms [33]. When controlling for depression, there were no 
significant differences found between PCL-M and PCL-S scores in this sample $(p>0.05)$.

For analyses involving PTSD symptom clusters, we used the emotional numbing four-factor model [25]. The model has reexperiencing (B1-B5) and hyperarousal (D1-D5) clusters identical to those in the DSM-IV [34]. However, this model partitions the DSM-IV avoidance symptoms into two separate clusters: an avoidance cluster $(\mathrm{C} 1-\mathrm{C} 2)$ and an emotional numbing cluster $(\mathrm{C} 3-\mathrm{C} 7)$, which primarily captures negative mood. The model has been supported in a large meta-analytic study [35].

Research has demonstrated that the PCL has excellent convergent validity with other measures of PTSD (Rs range from 0.77 to 0.93) [36]. Additionally, the PCL has strong test-retest reliability and internal consistency [36]. Internal consistency for the PCL total score in the current sample was good $(\alpha=0.90)$, and the reexperiencing $(\alpha=0.84)$, numbing $(\alpha=0.73)$, and hyperarousal $(\alpha=$ $0.76)$ clusters all evidenced acceptable reliability. The internal consistency of the avoidance cluster $(\alpha=0.65)$ was somewhat low, likely because the cluster is only composed of two items.

\section{Depression}

\section{Structured Clinical Interview for DSM-IV Axis I}

Disorders. The Structured Clinical Interview for DSMIV Axis I Disorders (SCID) is a clinician-administered diagnostic interview for DSM-IV Axis I disorders [37] and has strong agreement with multi-informant based diagnoses [38]. In the parent study, an abbreviated version of the SCID was given, and only data on diagnosis of current MDD (i.e., currently experiencing a major depressive episode) were used in the present study. The rate of comorbid MDD in this sample (33\%) was smaller than the reported rate of a recent meta-analysis [2], possibly a result of stringent application of the DSM-IV criteria.

Beck Depression Inventory-II. Depressive symptom severity was assessed for post hoc analyses using the BDIII [39], a 21-item self-report inventory of depression symptoms. Items are rated on a 4-point Likert scale, and higher total scale scores are indicative of greater symptom severity. The BDI-II has been used frequently in Veteran samples [40] and possesses strong validity and reliability [39]. Internal consistency of the BDI-II total score was good $(\alpha=0.93)$.
Quality of Life

Quality of life was assessed across the domains of social-material conditions, functioning, and satisfaction [18]. Quality of life related to social-material conditions was operationalized in this study as marital status and employment income, both of which came from the parent study's demographic questionnaire. Data on employment income were drawn from an open-ended question inquiring about current sources of income. For this study's analyses, we defined these domains using dichotomous variables (i.e., married/not married; employment income/ no employment income).

12-Item Short-Form Health Survey. Functioning was assessed using the 12-Item Short-Form Health Survey (SF-12), version 1 [41], a brief 12-item measure that uses both dichotomous and Likert-scale items to assess role functioning and health-related quality of life. The SF-12 asks questions about physical health limitations, the degree to which pain interferes with activities, and the impact of emotional problems on activities during the past month. The measure produces two summary scores, the SF-12 Physical Component Summary (PCS) and the SF-12 Mental Component Summary (MCS), which respectively assess the degree to which physical health and mental health problems have interfered with role functioning. The SF-12 has good reliability and validity [41] and has been used in military samples [42].

Quality of Life Inventory. Satisfaction was assessed using the Quality of Life Inventory (QOLI) [16], a psychometrically valid and reliable 32-item self-report inventory that assesses subjective life quality across 16 areas of life [43]. Each area of life on the QOLI is measured with two items: a 3-point Likert scale that assesses importance and a 6-point Likert scale that assesses satisfaction. Importance and satisfaction ratings are multiplied to produce a weighted satisfaction score for each area of life.

For this study, we organized areas of life assessed by the QOLI into four domains that have been supported in a large factor analytic study with a male Veteran sample [9]. These domains include achievement, self-expression, relationships, and surroundings. The achievement domain consists of the areas of health, self-esteem, goals and values, money, and work. The self-expression domain is composed of play, creativity, and learning areas of life, while the relationships domain consists of helping, love, friends, children, and relatives. Finally, the surroundings domain contains home, neighborhood, and community areas of life. Weighted satisfaction scores for the areas of life were 
averaged across each domain to produce a domain score, a metric with good internal consistency [24]. In the current study, the internal consistency across all domain scores was acceptable (achievement, $\alpha=0.81$; self-expression, $\alpha=0.76$; relationships, $\alpha=0.76$; and surroundings, $\alpha=$ $0.79)$.

\section{Statistical Analyses}

To determine whether Veterans with comorbid PTSD and depression demonstrated worse quality of life than those with PTSD only, we conducted a series of $t$-tests, chi-square tests, and regressions and examined point estimates and 95 percent confidence intervals (CIs) to evaluate significance. First, $t$-tests and chi-square tests were conducted between comorbid PTSD-MDD and PTSDonly groups across quality of life indicators, including marital status and employment income, the PCS and MCS scales, and the four QOLI domains (achievement, self-expression, relationships, and surroundings). For all quality of life indicators for which the comorbid PTSDMDD group was associated with significantly worse quality of life than the PTSD-only group, subsequent analyses were conducted to better understand the unique contributions of PTSD and depression. Each indicator was separately regressed on to each PTSD symptom cluster (reexperiencing, avoidance, numbing, and hyperarousal). Then, in cases in which a PTSD cluster was significantly and negatively related to the quality of life indicator, we conducted a series of simultaneous regressions. We first regressed the quality of life indicator on the PTSD cluster and comorbid MDD simultaneously to gauge whether inclusion of both the cluster and comorbid MDD would attenuate either variable's significant relationship with the quality of life indicator. We then regressed the quality of life indicator on to all the PTSD clusters simultaneously to determine whether the relationship remained significant when accounting for other PTSD symptoms. Finally, we regressed the quality of life indicator on to all PTSD symptom clusters and comorbid MDD simultaneously, and compared this model's $R^{2}$ value to the $R^{2}$ of the regression not including comorbid MDD. By doing this, we were able to examine the unique variance accounted for by all the PTSD clusters, as well as the incremental variance accounted for by comorbid MDD.

Initial findings involving satisfaction-related quality of life indicators (i.e., the QOLI domains) suggested the possibility that differences in reporter (self-report versus clinician) and level of measurement (categorical versus dimensional) across PTSD and depression measures potentially influenced findings. As such, we conducted post hoc analyses to specifically address the question of whether method variance affected study results. This included repeating all satisfaction-related quality of life regression analyses using the self-report, dimensional BDI-II total score to measure depression.

All regressions were conducted with MPlus, version 6.1 (Muthén \& Muthén; Los Angeles, California), using maximum likelihood estimation to handle missing data (8.27\%). Many independent variables in this study were converted to single-item latent variables, not to account for measurement error but only for the purposes of addressing missing data; this technique does not change interpretation of model parameters [44]. Descriptive statistics, $t$-tests and chi-square tests, and reliabilities were calculated using SPSS, version 19 (IBM Corp; Armonk, New York), and 95 percent CIs were calculated using SPSS and Microsoft Excel 2010, version 14.0.7 (Microsoft Corp; Redmond, Washington).

\section{RESULTS}

\section{Prediction of Quality of Life by Comorbid MDD}

Table 1 presents descriptive statistics for the comorbid PTSD-MDD and PTSD-only groups, as well as results from $t$-tests and chi-square tests. When compared with Veterans with PTSD only, Veterans with comorbid PTSD-MDD evidenced significantly worse satisfactionrelated quality of life on all four QOLI domains (achievement, self-expression, relationships, and surroundings). There were no significant differences across groups for either social-material conditions (marital status, employment income) or functioning (PCS and MCS scales).

\section{Separate and Simultaneous Regressions Involving PTSD Symptom Clusters}

The top portions of Tables 2 and $\mathbf{3}$ contain standardized results from separate regressions between each PTSD symptom cluster and each QOLI domain. All four PTSD symptom clusters were significantly and negatively associated with the achievement and self-expression domains, and the avoidance, numbing, and hyperarousal clusters were significantly negatively associated with the relationships domain. Numbing was the only cluster significantly and negatively associated with the surroundings domain. 
Table 1.

Descriptive statistics and $t$-test and chi-square test results for differences in quality of life indicators across comorbid PTSD-MDD and PTSD only groups. Data presented as $n(\%)$ or mean \pm standard deviation.

\begin{tabular}{|c|c|c|c|c|c|c|c|}
\hline Domain & $\begin{array}{l}\text { PTSD-MDD } \\
(n=49)\end{array}$ & $\begin{array}{c}\text { PTSD Only } \\
(n=99)\end{array}$ & $\chi^{2}$ & $t$ & $d f$ & CI Lower & CI Upper \\
\hline \multicolumn{8}{|c|}{ Social-Material Conditions } \\
\hline Employment Income & $4(8.2)$ & $12(12.1)$ & 0.53 & - & 1 & - & - \\
\hline Married & $31(63.3)$ & $55(55.6)$ & 0.80 & & 1 & - & - \\
\hline \multicolumn{8}{|l|}{ Functioning } \\
\hline PCS & $42.06 \pm 11.13$ & $43.43 \pm 12.04$ & - & 0.64 & 133 & -2.87 & 5.61 \\
\hline MCS & $38.97 \pm 12.52$ & $36.00 \pm 11.68$ & - & -1.36 & 133 & -7.30 & 1.34 \\
\hline \multicolumn{8}{|l|}{ Satisfaction } \\
\hline Achievement & $-0.85 \pm 2.30$ & $0.57 \pm 2.32$ & - & $3.32^{*}$ & 128 & 0.57 & 2.27 \\
\hline Self-Expression & $-0.23 \pm 1.93$ & $0.92 \pm 2.26$ & - & $2.90^{*}$ & 131 & 0.37 & 1.93 \\
\hline Relationships & $0.71 \pm 2.36$ & $1.63 \pm 1.95$ & - & $2.37^{\dagger}$ & 129 & 0.15 & 1.68 \\
\hline Surroundings & $0.56 \pm 2.68$ & $1.44 \pm 2.25$ & - & $1.99^{\dagger}$ & 131 & 0.01 & 1.75 \\
\hline $\begin{array}{l}\text { Note: Total sample size for } \\
\text { included in some analyses. } \\
{ }^{*} p<0.01 . \\
{ }^{\dagger} p<0.05 \text {. } \\
\text { Achievement }=\text { QOLI achie } \\
\text { Component Summary, MDI } \\
\text { Inventory, Relationships }=0\end{array}$ & $\begin{array}{l}\text { er than sum of PTS } \\
\text { tticipant nonrespons } \\
\text { in, CI Lower }=95 \% \\
\text { ressive disorder, PC } \\
\text { ships domain, Self-E }\end{array}$ & $\begin{array}{l}\text { and PTSD-only g } \\
\text { values were based o } \\
\text { ace interval lower bo } \\
12 \text { Physical Compor } \\
\text { o = QOLI self-expr } \\
\end{array}$ & Uppe & $\begin{array}{l}\text { participants } \\
\text { izes. } \\
5 \% \text { confide } \\
\mathrm{SD}=\text { posttr } \\
\text { rroundings } \\
\end{array}$ & va & $\begin{array}{l}\text { r bound, MCS } \\
\text { order, QOLI = } \\
\text { lings domain. }\end{array}$ & $\begin{array}{l}\text { data who were } \\
=\text { SF-12 Mental } \\
\text { Quality of Life }\end{array}$ \\
\hline
\end{tabular}

The middle portions of Tables 2 and $\mathbf{3}$ display the standardized results from simultaneous regressions involving comorbid MDD and individual PTSD symptom clusters. For the achievement domain, all PTSD symptom clusters retained their significant negative associations when analyzed together with comorbid MDD, while comorbid MDD retained its significant negative associations when analyzed with the reexperiencing, avoidance, and hyperarousal clusters. Comorbid MDD lost its significant negative relationship with achievement when analyzed jointly with the numbing cluster. Analyses involving the self-expression domain produced a pattern of significant relationships identical to those of the achievement domain. In addition, comorbid MDD lost its significant negative association with the relationship domain when analyzed with PTSD clusters, and only the PTSD numbing cluster retained it significant negative association when analyzed with comorbid MDD. There were no significant associations in simultaneous analyses involving the surroundings domain.

The bottom portions of Tables 2 and $\mathbf{3}$ present standardized results from the regressions containing all PTSD symptom clusters regressed simultaneously. The only PTSD symptom cluster that retained significance was numbing, which remained significantly negatively associated with the achievement, self-expression, and relationship domains. These relations remained significant even when comorbid MDD was entered into the analyses; comorbid MDD itself evidenced no significant relationships. Examination of model $R^{2}$ values indicated that a significant amount of variance was accounted for in analyses involving the achievement, self-expression, and relationship domains, and the inclusion of comorbid MDD failed to substantially increase the amount of variance accounted for in analyses.

\section{Post Hoc Analyses}

Results from post hoc regression analyses using the BDI-II are displayed in Tables $\mathbf{4}$ and 5. There were significant negative relationships between the BDI-II and all satisfaction-related quality of life domains in the separate analyses. Simultaneous analyses revealed a striking pattern: the BDI-II retained almost all its significant relationships when regressed jointly with individual PTSD clusters, while the reexperiencing, avoidance, and hyperarousal clusters lost significance across all quality of life domains when analyzed together with the BDI-II. The PTSD numbing cluster, however, retained significant negative relationships with the achievement, self-expression, and relationship domains. Simultaneous regressions between all the PTSD clusters and the BDI-II produced similar findings: the BDIII was significantly and negatively related to the achievement and surroundings domains and both the BDI-II and 
Table 2.

Standardized results of separate and simultaneous regressions between PTSD symptom clusters, MDD, and achievement and self-expression domains.

\begin{tabular}{|c|c|c|c|c|c|c|c|c|}
\hline \multirow{2}{*}{ Symptom Cluster } & \multicolumn{4}{|c|}{ Achievement } & \multicolumn{4}{|c|}{ Self-Expression } \\
\hline & $\bar{\beta}$ & $\mathrm{SE}$ & CI Lower & CI Upper & $\bar{\beta}$ & SE & CI Lower & CI Upper \\
\hline \multicolumn{9}{|l|}{ Separate } \\
\hline Reexp & $-0.34^{*}$ & 0.08 & -0.50 & -0.18 & $-0.30^{*}$ & 0.08 & -0.46 & -0.14 \\
\hline Avoid & $-0.24^{\dagger}$ & 0.08 & -0.40 & -0.08 & $-0.28^{\dagger}$ & 0.08 & -0.44 & -0.12 \\
\hline Numb & $-0.42^{*}$ & 0.07 & -0.56 & -0.28 & $-0.42^{*}$ & 0.07 & -0.56 & -0.28 \\
\hline Hyper & $-0.32^{*}$ & 0.08 & -0.48 & -0.16 & $-0.27^{\dagger}$ & 0.08 & -0.43 & -0.11 \\
\hline \multicolumn{9}{|c|}{ Simultaneous: Ind. PTSD Cluster \& MDD } \\
\hline & $-0.27^{\dagger}$ & 0.09 & -0.45 & -0.09 & $-0.24^{\dagger}$ & 0.09 & -0.42 & -0.06 \\
\hline MDD & $-0.19^{\sharp}$ & 0.09 & -0.37 & -0.01 & -0.17 & 0.09 & -0.33 & -0.01 \\
\hline Avoid & $-0.17^{\ddagger}$ & 0.09 & -0.34 & -0.01 & $-0.22^{\dagger}$ & 0.08 & -0.38 & -0.06 \\
\hline MDD & $-0.23^{\dagger}$ & 0.08 & -0.39 & -0.07 & $-0.18^{\ddagger}$ & 0.08 & -0.34 & -0.02 \\
\hline Numb & $-0.36^{*}$ & 0.08 & -0.52 & -0.20 & $-0.38^{*}$ & 0.08 & -0.54 & -0.22 \\
\hline MDD & -0.14 & 0.09 & -0.32 & 0.04 & -0.10 & 0.08 & -0.26 & 0.06 \\
\hline Hyper & $-0.26^{\dagger}$ & 0.08 & -0.42 & -0.10 & $-0.21^{\ddagger}$ & 0.09 & -0.39 & -0.03 \\
\hline MDD & $-0.20^{\ddagger}$ & 0.08 & -0.36 & -0.04 & $-0.18^{\ddagger}$ & 0.08 & -0.34 & -0.02 \\
\hline \multicolumn{9}{|c|}{ Simultaneous: All PTSD Clusters } \\
\hline & $0.20^{\dagger}$ & 0.06 & 0.08 & 0.32 & $0.19^{\dagger}$ & 0.06 & 0.07 & 0.31 \\
\hline Reexp & -0.15 & 0.11 & -0.37 & 0.07 & -0.07 & 0.11 & -0.29 & 0.15 \\
\hline Avoid & 0.05 & 0.11 & -0.17 & 0.27 & -0.08 & 0.11 & -0.30 & 0.14 \\
\hline Numb & $-0.32^{\dagger}$ & 0.10 & -0.52 & -0.12 & $-0.36^{*}$ & 0.09 & -0.54 & -0.18 \\
\hline Hyper & -0.09 & 0.11 & -0.31 & 0.13 & 0.03 & 0.11 & -0.19 & 0.25 \\
\hline \multicolumn{9}{|c|}{ Simultaneous: All PTSD Clusters \& MDD } \\
\hline & $0.21^{\dagger}$ & 0.06 & 0.09 & 0.33 & $0.19^{\dagger}$ & 0.06 & 0.07 & 0.31 \\
\hline Reexp & -0.13 & 0.11 & -0.35 & 0.09 & -0.06 & 0.11 & -0.28 & 0.16 \\
\hline Avoid & 0.06 & 0.11 & -0.16 & 0.28 & -0.07 & 0.11 & -0.29 & 0.15 \\
\hline Numb & $-0.29^{\dagger}$ & 0.10 & -0.49 & -0.09 & $-0.34^{\dagger}$ & 0.10 & -0.54 & -0.14 \\
\hline Hyper & -0.08 & 0.11 & -0.30 & 0.14 & 0.03 & 0.11 & -0.19 & 0.25 \\
\hline MDD & -0.12 & 0.09 & -0.30 & 0.06 & -0.09 & 0.09 & -0.27 & 0.09 \\
\hline
\end{tabular}

${ }^{*} p<0.001$.

$\dot{t} p<0.01$.

$\$ p<0.05$.

Achievement $=$ QOLI achievement domain, Avoid $=$ PTSD avoidance cluster, CI Lower $=95 \%$ confidence interval lower bound, CI Upper $=95 \%$ confidence interval upper bound, Hyper = PTSD hyperarousal cluster, Ind. = individual, $\mathrm{MDD}=$ major depressive disorder, Numb $=$ PTSD numbing cluster, $\mathrm{PTSD}=$ posttraumatic stress disorder, QOLI $=$ Quality of Life Inventory, Reexp $=$ PTSD reexperiencing cluster, SE $=$ standard error, Self-Expression $=$ QOLI self-expression domain

PTSD numbing cluster had significant negative associations with the self-expression and relationship domains. There were no significant relationships between other PTSD clusters and quality of life domains in these simultaneous analyses. Results represent small effects for predicting quality of life outcomes [45].

\section{DISCUSSION}

Previous research has demonstrated that PTSD can have a negative impact on quality of life for Veterans [6] and that the presence of comorbid depression can lead to further life quality problems [14]. The current study adds to this literature by exploring the impact of comorbid depression on social-material conditions, functioning, and satisfaction-related quality of life and the unique contributions of each PTSD symptom cluster and comorbid depression to quality of life impairment in a sample of male combat Veterans with PTSD.

Our hypotheses were partially confirmed, as our study supported past research documenting, in individuals with PTSD, the detrimental impact of comorbid depression on satisfaction with relationships and the surrounding 
JRRD, Volume 52, Number 5, 2015

Table 3.

Standardized results of separate and simultaneous regressions between PTSD symptom clusters, MDD, and relationships and surroundings domains.

\begin{tabular}{|c|c|c|c|c|c|c|c|c|}
\hline \multirow{2}{*}{ Symptom Cluster } & \multicolumn{4}{|c|}{ Relationships } & \multicolumn{4}{|c|}{ Surroundings } \\
\hline & $\bar{\beta}$ & SE & CI Lower & CI Upper & $\bar{\beta}$ & SE & CI Lower & CI Upper \\
\hline \multicolumn{9}{|l|}{ Separate } \\
\hline Reexp & -0.16 & 0.09 & -0.34 & 0.02 & -0.12 & 0.09 & -0.30 & 0.06 \\
\hline Avoid & & 0.09 & -0.39 & -0.03 & -0.11 & 0.09 & -0.29 & 0.07 \\
\hline Numb & & 0.07 & -0.55 & -0.27 & $-0.17^{*}$ & 0.08 & -0.33 & -0.01 \\
\hline Hyper & & 0.09 & -0.40 & -0.04 & -0.14 & 0.09 & -0.32 & 0.04 \\
\hline \multicolumn{9}{|c|}{ Simultaneous: Ind. PTSD Cluster \& MDD } \\
\hline Reexp & & - & - & - & - & - & - & - \\
\hline MDD & & - & - & - & - & - & - & - \\
\hline Avoid & & 0.09 & -0.34 & 0.02 & - & - & - & - \\
\hline MDD & & 0.09 & -0.34 & 0.02 & - & - & - & - \\
\hline Numb & & 0.08 & -0.55 & -0.23 & -0.11 & 0.09 & -0.29 & 0.07 \\
\hline MDD & & 0.09 & -0.23 & 0.13 & -0.13 & 0.09 & -0.31 & 0.05 \\
\hline Hyper & & 0.09 & -0.35 & 0.01 & - & - & - & - \\
\hline MDD & & 0.09 & -0.34 & 0.02 & - & - & - & - \\
\hline \multicolumn{9}{|c|}{ Simultaneous: All PTSD Clusters } \\
\hline$R^{2}$ & & 0.06 & 0.05 & 0.29 & 0.03 & 0.03 & -0.03 & 0.09 \\
\hline Reexp & & 0.11 & -0.13 & 0.31 & 0.01 & 0.12 & -0.23 & 0.25 \\
\hline Avoid & & 0.11 & -0.28 & 0.16 & -0.02 & 0.12 & -0.26 & 0.22 \\
\hline Numb & & 0.10 & -0.64 & -0.24 & -0.13 & 0.11 & -0.35 & 0.09 \\
\hline Hyper & & 0.12 & -0.22 & 0.26 & -0.05 & 0.12 & -0.29 & 0.19 \\
\hline \multicolumn{9}{|c|}{ Simultaneous: All PTSD Clusters \& MDD } \\
\hline$R^{2}$ & & 0.06 & 0.06 & 0.30 & - & - & - & - \\
\hline Reexp & & 0.11 & -0.12 & 0.32 & - & - & - & - \\
\hline Avoid & & 0.11 & -0.28 & 0.16 & - & - & - & - \\
\hline Numb & & 0.10 & -0.62 & -0.22 & - & - & - & - \\
\hline Hyper & & 0.12 & -0.22 & 0.26 & - & - & - & - \\
\hline MDD & -0.07 & 0.09 & -0.25 & 0.11 & - & - & - & - \\
\hline
\end{tabular}

Note: Dash indicates that value was not computed.

${ }^{*} p<0.05$.

${ }^{\dagger} p<0.001$

$\$ p<0.01$.

Avoid $=$ PTSD avoidance cluster, CI Lower $=95 \%$ confidence interval lower bound, CI Upper $=95 \%$ confidence interval upper bound, Hyper $=$ PTSD hyperarousal cluster, Ind. = individual, $\mathrm{MDD}=$ major depressive disorder, Numb $=$ PTSD numbing cluster, PTSD = posttraumatic stress disorder, QOLI $=$ Quality of Life Inventory, Reexp $=$ PTSD reexperiencing cluster, Relationships $=$ QOLI relationships domain, $\mathrm{SE}=$ standard error, Surroundings $=$ QOLI surroundings domain.

environment [15] and expanded upon past research by finding negative relationships with achievement and self-expression domains. However, we also found that comorbid depression was not significantly related to social-material condition-related quality of life, nor was it associated with diminished role functioning, the latter of which is in contrast to existing research [12]. When exploring the unique contributions of depression and PTSD to reduced quality of life, PTSD numbing symptoms initially seemed to account for the association with decreased quality of life across all satisfaction-related domains. However, a very different pattern of findings emerged in post hoc analyses when operationalizing depression using the self-report, dimensional BDI-II instead of the clinician-administered, categorical MDD SCID diagnosis. Comorbid depression symptoms had more negative associations with decreased quality of life and constituted the sole significant predictor of quality of life in the achievement and surrounding domains when considered jointly with all PTSD clusters. Furthermore, the BDI-II also predicted decreased quality of life in the selfexpression and relationship domains, although these were 
Table 4.

Standardized results of separate and simultaneous regressions between PTSD symptom clusters, BDI-II, and achievement and self-expression domains.

\begin{tabular}{|c|c|c|c|c|c|c|c|c|}
\hline \multirow{2}{*}{ Symptom Cluster } & \multicolumn{4}{|c|}{ Achievement } & \multicolumn{4}{|c|}{ Self-Expression } \\
\hline & $\boldsymbol{\beta}$ & SE & CI Lower & CI Upper & $\boldsymbol{\beta}$ & SE & CI Lower & CI Upper \\
\hline \multicolumn{9}{|l|}{ Separate } \\
\hline BDI-II & $-0.52^{*}$ & 0.06 & -0.64 & -0.40 & $-0.42^{*}$ & 0.07 & -0.56 & -0.28 \\
\hline \multicolumn{9}{|c|}{ Simultaneous: Ind. PTSD Cluster \& BDI-II } \\
\hline Reexp & -0.09 & 0.09 & -0.27 & 0.09 & -0.11 & 0.09 & -0.29 & 0.07 \\
\hline BDI-II & $-0.47^{*}$ & 0.08 & -0.63 & -0.31 & $-0.36^{*}$ & 0.09 & -0.54 & -0.18 \\
\hline Avoid & -0.02 & 0.08 & -0.18 & 0.14 & -0.11 & 0.09 & -0.29 & 0.07 \\
\hline BDI-II & $-0.51^{*}$ & 0.07 & -0.65 & -0.37 & $-0.37^{*}$ & 0.08 & -0.53 & -0.21 \\
\hline Numb & $-0.18^{\dagger}$ & 0.09 & -0.36 & -0.01 & -0.28 & 0.09 & -0.46 & -0.10 \\
\hline BDI-II & $-0.40^{*}$ & 0.09 & -0.58 & -0.22 & $-0.24^{\dagger}$ & 0.10 & -0.44 & -0.04 \\
\hline Hyper & -0.13 & 0.08 & -0.29 & 0.03 & -0.11 & 0.09 & -0.29 & 0.07 \\
\hline BDI-II & $-0.46^{*}$ & 0.07 & -0.60 & -0.32 & $-0.37^{*}$ & 0.08 & -0.53 & -0.21 \\
\hline \multicolumn{9}{|c|}{ Simultaneous: All PTSD Clusters \& BDI-II } \\
\hline & $0.30^{*}$ & 0.07 & 0.16 & 0.44 & $0.22^{\ddagger}$ & 0.06 & 0.10 & 0.34 \\
\hline Reexp & -0.04 & 0.11 & -0.26 & 0.18 & -0.02 & 0.11 & -0.24 & 0.20 \\
\hline Avoid & 0.09 & 0.10 & -0.11 & 0.29 & -0.05 & 0.11 & -0.27 & 0.17 \\
\hline Numb & -0.15 & 0.10 & -0.35 & 0.05 & $-0.26^{\dagger}$ & 0.10 & -0.46 & -0.06 \\
\hline Hyper & -0.10 & 0.10 & -0.30 & 0.10 & 0.02 & 0.11 & -0.20 & 0.24 \\
\hline BDI-II & $-0.40^{*}$ & 0.09 & -0.58 & -0.22 & $-0.23^{\dagger}$ & 0.10 & -0.43 & -0.03 \\
\hline
\end{tabular}

${ }^{*} p<0.001$.

${ }^{\dagger} p<0.05$.

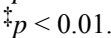

Achievement $=$ QOLI achievement domain, Avoid $=$ PTSD avoidance cluster, BDI-II $=$ Beck Depression Inventory-II total score, CI Lower $=95 \%$ confidence interval lower bound, CI Upper $=95 \%$ confidence interval upper bound, Hyper $=$ PTSD hyperarousal cluster, Ind. $=$ individual, Numb $=$ PTSD numbing cluster, $\mathrm{PTSD}=$ posttraumatic stress disorder, QOLI $=$ Quality of Life Inventory, Reexp $=$ PTSD reexperiencing cluster, SE $=$ standard error, Self-Expression $=$ QOLI selfexpression domain.

generally small effects. The only PTSD cluster that demonstrated significant relationships with these two domains was numbing, with small to medium-sized effects.

These findings were consistent with past research suggesting that depression has a stronger influence on decreased quality of life than does PTSD [20,22]. Past studies have also produced similar findings regarding the importance of the PTSD numbing cluster to quality of life in Veterans with PTSD $[9,18]$, and this study supports and extends those findings by suggesting that, of all the PTSD symptom clusters, numbing has the largest negative association with quality of life. Taken together, the results of this study suggest that, for Veterans with PTSD, satisfaction-related quality of life problems are in large part accounted for by negative affect.

Negative affect has been conceptualized as a higherorder general distress factor that subsumes many depressed and anxious mood-related symptoms, including those of depression and the PTSD numbing cluster, and may explain the high rates of comorbidity across disorders [46]. To address comorbidities, researchers have proposed an empirically-based reorganization of the DSM diagnostic categories to create a superclass encompassing all mood and anxiety disorders. Coined the Quadripartite Model, this overarching framework involves three subclasses, including fear disorders (agoraphobia, panic disorder, social phobia, and specific phobia), distress disorders (dysthymic disorder, generalized anxiety disorder, PTSD, and major depression), and bipolar disorders (bipolar I, bipolar II, and cyclothymia) [47]. Proponents of the Quadripartite Model advocate removing negative affect symptoms (i.e., numbing symptoms of PTSD) from diagnostic criteria and measuring them dimensionally, to both improve measurement and also to aid in differential diagnosis [46].

Although not ultimately endorsed by the DSM-5 categorization, the Quadripartite Model categorized PTSD as a distress disorder because of strong correlations with 
JRRD, Volume 52, Number 5, 2015

Table 5.

Standardized results of separate and simultaneous regressions between PTSD symptom clusters, BDI-II, and relationships and surroundings domains.

\begin{tabular}{|c|c|c|c|c|c|c|c|c|}
\hline \multirow{2}{*}{ Symptom Cluster } & \multicolumn{4}{|c|}{ Relationships } & \multicolumn{4}{|c|}{ Surroundings } \\
\hline & $\bar{\beta}$ & SE & CI Lower & CI Upper & $\bar{\beta}$ & SE & CI Lower & CI Upper \\
\hline \multicolumn{9}{|l|}{ Separate } \\
\hline BDI-II & $-0.37^{*}$ & 0.08 & -0.53 & -0.21 & $-0.30^{*}$ & 0.08 & -0.46 & -0.14 \\
\hline \multicolumn{9}{|c|}{ Simultaneous: Ind. PTSD Cluster \& BDI-II } \\
\hline Reexp & - & - & - & - & - & - & - & - \\
\hline BDI-II & - & - & - & - & - & - & - & - \\
\hline Avoid & -0.06 & 0.09 & -0.24 & 0.12 & - & - & - & - \\
\hline BDI-II & $-0.34^{*}$ & 0.09 & -0.52 & -0.16 & - & - & - & - \\
\hline Numb & $-0.31^{\dagger}$ & 0.10 & -0.51 & -0.11 & 0.01 & 0.10 & -0.19 & 0.21 \\
\hline BDI-II & -0.19 & 0.10 & -0.39 & 0.01 & $-0.29^{\dagger}$ & 0.10 & -0.49 & -0.09 \\
\hline Hyper & -0.07 & 0.09 & -0.25 & 0.11 & - & - & - & - \\
\hline BDI-II & $-0.34^{*}$ & 0.09 & -0.52 & -0.16 & - & - & - & - \\
\hline \multicolumn{9}{|c|}{ Simultaneous: All PTSD Clusters \& BDI-II } \\
\hline & $0.21^{\dagger}$ & 0.06 & 0.09 & 0.33 & 0.09 & 0.05 & -0.01 & 0.19 \\
\hline Reexp & 0.14 & 0.11 & -0.08 & 0.36 & 0.08 & 0.12 & -0.16 & 0.32 \\
\hline Avoid & -0.04 & 0.11 & -0.26 & 0.18 & 0.02 & 0.11 & -0.20 & 0.24 \\
\hline Numb & $-0.34^{\dagger}$ & 0.11 & -0.56 & -0.12 & 0.01 & 0.11 & -0.21 & 0.23 \\
\hline Hyper & 0.02 & 0.11 & -0.20 & 0.24 & -0.06 & 0.12 & -0.30 & 0.18 \\
\hline BDI-II & $-0.23^{t}$ & 0.10 & -0.43 & -0.03 & $-0.32^{\dagger}$ & 0.11 & -0.54 & -0.10 \\
\hline
\end{tabular}

Note: Dash indicates that value was not computed

${ }^{*} p<0.001$

${ }^{\dagger} p<0.01$.

$\$ p<0.05$.

Avoid $=$ PTSD avoidance cluster, BDI-II $=$ Beck Depression Inventory-II total score, CI Lower $=95 \%$ confidence interval lower bound, CI Upper $=95 \%$ confidence interval upper bound, Hyper $=$ PTSD hyperarousal cluster, Ind. = individual, Numb $=$ PTSD numbing cluster, PTSD $=$ posttraumatic stress disorder, $\mathrm{QOLI}=$ Quality of Life Inventory, Reexp $=$ PTSD reexperiencing cluster, Relationships $=$ QOLI relationships domain, SE $=$ standard error, Surroundings $=$ QOLI surroundings domain.

indicators of depression, and a substantial amount of negative affectivity variance [46]. This was largely due to the PTSD numbing symptoms, or alternately PTSD dysphoria symptoms, another highly supported factor structure that differs slightly from the numbing factor solution [48]. Analysis of large epidemiological samples shows that dysphoria and numbing symptoms are more highly correlated with depression than are other PTSD symptoms clusters (i.e., re-experiencing, avoidance, hyperarousal) [46]. The results of the current study further support the connections between PTSD numbing symptoms and comorbid depression by demonstrating their similar negative associations with quality of life.

However, it is important to note that PTSD numbing symptoms and comorbid depression did not have an identical pattern of significant findings across quality of life domains. Rather, in simultaneous analyses, comorbid depression as measured by the BDI-II was significantly negatively related to quality of life indicators when controlling for PTSD numbing symptoms, and PTSD numbing symptoms were significantly negatively related to quality of life indicators when controlling for the BDI-II. This is interesting in that it suggests that the manifestations of negative affect in PTSD numbing symptoms and comorbid depression were distinct enough to result in a differential pattern of associations with quality of life domains. Further empirical research is needed to examine the different manifestations of negative affect in the mood and anxiety disorders as well as their clinical correlates, especially when determining inclusion of items for a general measure of negative affect across disorders.

These results have significant clinical implications. Given the finding that comorbid depression and PTSD numbing symptoms were most strongly associated with 
negative satisfaction-related quality of life, results suggest that specifically targeting negative affect could result in improved satisfaction across a number of different quality of life domains. They also suggest that interventions targeting negative affect should be developed to augment evidence-based PTSD therapies. Research into one such intervention, which includes behavioral activation components alongside exposure therapy, found that the combined treatment lowered both PTSD symptoms and the overlapping symptoms of PTSD and depression [49]. While the researchers did not examine quality of life outcomes, given the robust empirical associations between both disorders and quality of life, it is conceivable that this innovative treatment modality could improve life quality for Veterans. Other approaches that could potentially address negative affect in Veterans with PTSD are transdiagnostic psychotherapies for mood and anxiety disorders [50]. Transdiagnostic treatments for the emotional disorders focus more broadly on the overarching symptoms found across all of the mood and anxiety disorders (e.g., poor emotional regulation) [51]; however, to date most studies have explored civilian samples with low rates of PTSD [52]. One protocol, Transdiagnostic Behavioral Therapy, was developed specifically for use with Veteran populations and has showed significant promise in reducing both PTSD and depression symptoms [52].

The findings of this study are also relevant to future research examining the clinical correlates of PTSD and comorbid depression. They suggest that it is important to assess depression using categorical and dimensional measures as well as multiple reporters. Additionally, results indicate that it is necessary to explore associations with specific PTSD symptom clusters, analyzed both individually and simultaneously. Given that the DSM-5 expanded the role of negative affect in the diagnosis of PTSD through the creation of the negative alterations in cognitions and mood clusters, these considerations are even more pertinent when exploring the clinical correlates of PTSD and comorbid depression.

This study has several limitations that should be noted. The $R^{2}$ values of significant simultaneous regression analyses indicated that comorbid PTSD and depression only accounted for a portion of the variance across quality of life scores. Relatedly, effect size estimates were generally in the small range, and the risk of type I error was elevated due to the number of statistical procedures conducted. These limitations highlight the need for further research into predictors of satisfaction-related quality of life using both independent and larger samples. Also, the BDI-II and diagnosis of MDD produced disparate findings in this sample. This is possibly a result of method variance, as the continuous BDI-II may have captured a larger degree of negative affect variance than the dichotomous MDD diagnosis, which may then have resulted in a stronger association with quality of life indicators. Additional research is needed using multiple self-report and clinician-administered measures, across both continuous and dichotomous predictors, to explore this issue. All measures of quality of life in this study, including objective measures, were obtained through self-report methodology. Use of other reporters, such as employers, spouses, and friends, could enhance the reliability of findings [53]. Furthermore, our study was cross-sectional in nature and therefore causality and temporal precedence cannot be conclusively established. Findings would be greatly enhanced by prospective, longitudinal research designs. Finally, the sample was limited in that it was all male and included only treatmentseeking Veterans.

\section{CONCLUSIONS}

This study found that combat Veterans with comorbid PTSD-MDD reported significantly worse satisfactionrelated quality of life than those with PTSD alone, although this finding was largely attributable to PTSD numbing symptoms. Subsequent analyses comparing the impact of numbing symptoms to dimensionally assessed depressive symptoms revealed that depression exerted a stronger influence, although numbing symptoms were still uniquely associated with poor quality of life. Together, these findings indicate the importance of assessing and treating negative affect in Veterans with PTSD and suggest the need to develop innovate treatment approaches to specifically target negative affect, such as the addition of behavioral activation to exposure therapy [49] or transdiagnostic treatment approaches [52]. Future research should examine the impact of negative affect on quality of life in other traumatized populations, as well as the impact of comorbid depression on quality of life outcomes following evidence-based PTSD treatment.

\section{ACKNOWLEDGMENTS}

\section{Author Contributions:}

Study concept and design: P. A. Raab, M. Mackintosh, D. F. Gros. 
Acquisition of data: P. A. Raab, M. Mackintosh, L. A. Morland. Analysis and interpretation of data: P. A. Raab, M. Mackintosh, D. F. Gros.

Drafting of manuscript: P. A. Raab. Critical revision of manuscript for important intellectual content: M. Mackintosh, D. F. Gros, L. A. Morland.

Financial Disclosures: The authors have declared that no competing interests exist.

Funding/Support: This material was based on work supported in part by the VA Office of Research and Development, Health Services Research \& Development Service (grant DHI 07-259) and the Department of Defense, Congressionally Directed Medical Research Programs (grant PT074516). Support was also provided by the VA National Center for PTSD and with resources and the use of facilities at Spark M. Matsunaga Medical Center, VA Pacific Islands Health Care System.

Additional Contributions: Leslie A. Morland, PsyD, is now with the VA San Diego Healthcare System, San Diego, California. Phillip A. Raab, PhD, is now with the VA Pittsburgh Healthcare System, Pittsburgh, Pennsylvania.

Institutional Review: This research was approved by the VA Pacific Islands Health Care System Institutional Review Board, and all participants provided written informed consent prior to participating.

Participant Follow-Up: The authors do not plan to notify study participants of publication of this article.

Disclaimer: All views and opinions expressed herein are those of the authors and do not necessarily reflect those of our respective institutions or the VA.

\section{REFERENCES}

1. Gros DF, Price M, Magruder KM, Frueh BC. Symptom overlap in posttraumatic stress disorder and major depression. Psychiatry Res. 2012;196(2-3):267-70.

[PMID:22386220]

http://dx.doi.org/10.1016/j.psychres.2011.10.022

2. Rytwinski NK, Scur MD, Feeny NC, Youngstrom EA. The co-occurrence of major depressive disorder among individuals with posttraumatic stress disorder: A meta-analysis. J Trauma Stress. 2013;26(3):299-309. [PMID:23696449] http://dx.doi.org/10.1002/jts.21814

3. O'Donnell ML, Creamer M, Pattison P. Posttraumatic stress disorder and depression following trauma: Understanding comorbidity. Am J Psychiatry. 2004;161(8):1390-96.

[PMID:15285964] http://dx.doi.org/10.1176/appi.ajp.161.8.1390

4. Gros DF, Simms LJ, Acierno R. Specificity of posttraumatic stress disorder symptoms: An investigation of comorbidity between posttraumatic stress disorder symptoms and depression in treatment-seeking veterans. J Nerv Ment Dis. 2010;198(12):885-90. [PMID:21135640] http://dx.doi.org/10.1097/NMD.0b013e3181fe7410

5. World Health Organization. Constitution of the World Health Organization. Geneva (Switzerland): WHO; 1948.
6. Magruder KM, Frueh BC, Knapp RG, Johnson MR, Vaughan JA 3rd, Carson TC, Powell DA, Hebert R. PTSD symptoms, demographic characteristics, and functional status among veterans treated in VA primary care clinics. J Trauma Stress. 2004;17(4):293-301. [PMID:15462536] http://dx.doi.org/10.1023/B:JOTS.0000038477.47249.c8

7. Rona RJ, Jones M, Iversen A, Hull L, Greenberg N, Fear NT, Hotopf M, Wessely S. The impact of posttraumatic stress disorder on impairment in the UK military at the time of the Iraq war. J Psychiatr Res. 2009;43(6):649-55.

[PMID:18950801]

http://dx.doi.org/10.1016/j.jpsychires.2008.09.006

8. Breslau N, Lucia VC, Davis GC. Partial PTSD versus full PTSD: An empirical examination of associated impairment. Psychol Med. 2004;34(7):1205-14. [PMID:15697047] http://dx.doi.org/10.1017/S0033291704002594

9. Lunney CA, Schnurr PP. Domains of quality of life and symptoms in male veterans treated for posttraumatic stress disorder. J Trauma Stress. 2007;20(6):955-64. [PMID:18157892] http://dx.doi.org/10.1002/jts.20269

10. Elbogen EB, Johnson SC, Wagner HR, Newton VM, Beckham JC. Financial well-being and postdeployment adjustment among Iraq and Afghanistan war veterans. Mil Med. 2012;177(6):669-75. [PMID:22730842] http://dx.doi.org/10.7205/MILMED-D-11-00388

11. Rapaport MH, Clary C, Fayyad R, Endicott J. Quality-oflife impairment in depressive and anxiety disorders. Am J Psychiatry. 2005;162(6):1171-78. [PMID:15930066] http://dx.doi.org/10.1176/appi.ajp.162.6.1171

12. Blanchard EB, Buckley TC, Hickling EJ, Taylor AE. Posttraumatic stress disorder and comorbid major depression: Is the correlation an illusion? J Anxiety Disord. 1998; 12(1):21-37. [PMID:9549607] http://dx.doi.org/10.1016/S0887-6185(97)00047-9

13. Momartin S, Silove D, Manicavasagar V, Steel Z. Comorbidity of PTSD and depression: Associations with trauma exposure, symptom severity and functional impairment in Bosnian refugees resettled in Australia. J Affect Disord. 2004;80(2-3):231-38. [PMID:15207936] http://dx.doi.org/10.1016/S0165-0327(03)00131-9

14. Ginzburg K, Ein-Dor T, Solomon Z. Comorbidity of posttraumatic stress disorder, anxiety and depression: A 20year longitudinal study of war veterans. J Affect Disord. 2010;123(1-3):249-57. [PMID:19765828] http://dx.doi.org/10.1016/j.jad.2009.08.006

15. Ikin JF, Creamer MC, Sim MR, McKenzie DP. Comorbidity of PTSD and depression in Korean War veterans: Prevalence, predictors, and impairment. J Affect Disord. 2010; 125(1-3):279-86. [PMID:20071032] http://dx.doi.org/10.1016/j.jad.2009.12.005

16. Frisch MB. Quality of Life Inventory: Manual and treatment guide. Minneapolis (MN): NCS Pearson and Pearson Assessments; 1994. 
17. Gladis MM, Gosch EA, Dishuk NM, Crits-Christoph P. Quality of life: Expanding the scope of clinical significance. J Consult Clin Psychol. 1999;67(3):320-31. [PMID:10369052] http://dx.doi.org/10.1037/0022-006X.67.3.320

18. Schnurr PP, Lunney CA, Bovin MJ, Marx BP. Posttraumatic stress disorder and quality of life: Extension of findings to veterans of the wars in Iraq and Afghanistan. Clin Psychol Rev. 2009;29(8):727-35. [PMID:19744758] http://dx.doi.org/10.1016/j.cpr.2009.08.006

19. Mogotsi M, Kaminer D, Stein DJ. Quality of life in the anxiety disorders. Harv Rev Psychiatry. 2000;8(6):273-82. [PMID:11133822] http://dx.doi.org/10.1080/hrp.8.6.273

20. Pittman JO, Goldsmith AA, Lemmer JA, Kilmer MT, Baker DG. Post-traumatic stress disorder, depression, and health-related quality of life in OEF/OIF veterans. Qual Life Res. 2012;21(1):99-103. [PMID:21516356] http://dx.doi.org/10.1007/s11136-011-9918-3

21. Rauch SA, Favorite T, Giardino N, Porcari C, Defever E, Liberzon I. Relationship between anxiety, depression, and health satisfaction among veterans with PTSD. J Affect Disord. 2010;121(1-2):165-68. [PMID:19535152] http://dx.doi.org/10.1016/j.jad.2009.05.026

22. Aversa LH, Stoddard JA, Doran NM, Au S, Chow B, McFall M, Saxon A, Baker DG. PTSD and depression as predictors of physical health-related quality of life in tobacco-dependent veterans. J Psychosom Res. 2012;73(3): 185-90. [PMID:22850258]

23. Kuhn E, Blanchard EB, Hickling EJ. Posttraumatic stress disorder and psychosocial functioning within two samples of MVA survivors. Behav Res Ther. 2003;41(9):1105-12. [PMID:12914811] http://dx.doi.org/10.1016/S0005-7967(03)00071-8

24. Schnurr PP, Lunney CA. Exploration of gender differences in how quality of life relates to posttraumatic stress disorder in male and female veterans. J Rehabil Res Dev. 2008; 45(3):383-93. [PMID:18629747] http://dx.doi.org/10.1682/JRRD.2007.06.0099

25. King DW, Leskin GA, King LA, Weathers FW. Confirmatory factor analysis of the clinician-administered PTSD Scale: Evidence for the dimensionality of posttraumatic stress disorder. Psychol Assess. 1998;10(2):90-96. http://dx.doi.org/10.1037/1040-3590.10.2.90

26. American Psychiatric Association. Diagnostic and statistical manual of mental disorders. 5th ed. Arlington (VA): American Psychiatric Publishing; 2013.

27. Resick PA, Monson CM, Chard KM. Cognitive processing therapy: Veteran/military version. Washington (DC): Department of Veterans Affairs; 2007.

28. Morland LA, Mackintosh MA, Greene CJ, Rosen CS, Chard KM, Resick P, Frueh BC. Cognitive processing therapy for posttraumatic stress disorder delivered to rural veterans via telemental health: A randomized noninferiority clinical trial. J Clin Psychiatry. 2014;75(5):470-76.

[PMID:24922484]

http://dx.doi.org/10.4088/JCP.13m08842

29. Morland LA, Hynes AK, Mackintosh MA, Resick PA, Chard KM. Group cognitive processing therapy delivered to veterans via telehealth: A pilot cohort. J Trauma Stress. 2011;24(4):465-69. [PMID:21793047] http://dx.doi.org/10.1002/jts.20661

30. Weathers FW, Ruscio AM, Keane TM. Psychometric properties of nine scoring rules for the Clinician-Administered Posttraumatic Stress Disorder Scale. Psychol Assess. 1999;11(2):124-33. http://dx.doi.org/10.1037/1040-3590.11.2.124

31. Blake DD, Weathers FW, Nagy LM, Kaloupek DG, Gusman FD, Charney DS, Keane TM. The development of a clinician-administered PTSD scale. J Trauma Stress. 1995; 8(1):75-90. [PMID:7712061] http://dx.doi.org/10.1002/jts.2490080106

32. Weathers FW, Litz BT, Herman DS, Huska JA, Keane TM, editors. The PTSD Checklist (PCL): Reliability, validity, and diagnostic utility. Annual Meeting of the International Society for Traumatic Stress Studies, San Antonio, TX; 1993.

33. Riviere LA, Edens EN, Adler AB, Bliese PD, Klocko RP, Hoge CW. Modifying instructions on the posttraumatic stress disorder checklist for military populations does not change symptom reporting. J Nerv Ment Dis. 2011;199(3): 199-202. [PMID:21346492] http://dx.doi.org/10.1097/NMD.0b013e31820caee4

34. American Psychiatric Association. Diagnostic and statistical manual of mental disorders. 4th ed. Text revision. Washington (DC): American Psychiatric Association; 2000.

35. Yufik T, Simms LJ. A meta-analytic investigation of the structure of posttraumatic stress disorder symptoms. J Abnorm Psychol. 2010;119(4):764-76. [PMID:21090877] http://dx.doi.org/10.1037/a0020981

36. Orsillo SM. Practitioner's guide to empirically based measures of anxiety. Chapter 19: Measures for acute stress disorder and posttraumatic stress disorder. New York (NY): Springer; 2002; p. 255-307.

37. First MB, Spitzer RL, Gibbon M, Williams JB. Structured clinical interview for DSM-IV-TR Axis I disorders: Research version. New York (NY): Biometrics Research, New York State Psychiatric Institute; 2002.

38. Ramirez Basco M, Bostic JQ, Davies D, Rush AJ, Witte B, Hendrickse W, Barnett V. Methods to improve diagnostic accuracy in a community mental health setting. Am J Psychiatry. 2000;157(10):1599-1605. [PMID:11007713] http://dx.doi.org/10.1176/appi.ajp.157.10.1599

39. Beck A, Steer R, Brown G. Beck Depression Inventory-II, Manual. San Antonio (TX): The Psychological Corporation; 1996. 
40. Walter KH, Barnes SM, Chard KM. The influence of comorbid MDD on outcome after residential treatment for veterans with PTSD and a history of TBI. J Trauma Stress. 2012;25(4):426-32. [PMID:22821663]

http://dx.doi.org/10.1002/jts.21722

41. Ware J Jr, Kosinski M, Keller SD. A 12-Item Short-Form Health Survey: Construction of scales and preliminary tests of reliability and validity. Med Care. 1996;34(3):220-33. [PMID:8628042] http://dx.doi.org/10.1097/00005650-199603000-00003

42. McKenzie DP, Ikin JF, McFarlane AC, Creamer M, Forbes AB, Kelsall HL, Glass DC, Ittak P, Sim MR. Psychological health of Australian veterans of the 1991 Gulf War: An assessment using the SF-12, GHQ-12 and PCL-S. Psychol Med. 2004;34(8):1419-30. [PMID:15724873]

http://dx.doi.org/10.1017/S0033291704002818

43. Frisch MB, Cornell J, Villanueva M, Retzlaff PJ. Clinical validation of the Quality of Life Inventory. A measure of life satisfaction for use in treatment planning and outcome assessment. Psychol Assess. 1992;4(1):92-101.

http://dx.doi.org/10.1037/1040-3590.4.1.92

44. Enders CK. Applied missing data analysis. New York (NY): Guilford Press; 2010.

45. Cohen J. Statistical power analysis for the behavioral sciences. 2nd ed. Hillsdale (NJ): Lawrence Erlbaum Associates; 1988.

46. Watson D. Differentiating the mood and anxiety disorders: A quadripartite model. Annu Rev Clin Psychol. 2009;5: 221-47. [PMID:19327030]

http://dx.doi.org/10.1146/annurev.clinpsy.032408.153510

47. Watson D, O'Hara MW, Stuart S. Hierarchical structures of affect and psychopathology and their implications for the classification of emotional disorders. Depress Anxiety. 2008;25(4):282-88. [PMID:18415951]

http://dx.doi.org/10.1002/da.20496

48. Simms LJ, Watson D, Doebbeling BN. Confirmatory factor analyses of posttraumatic stress symptoms in deployed and nondeployed veterans of the Gulf War. J Abnorm Psychol. 2002;111(4):637-47. [PMID:12428777] http://dx.doi.org/10.1037/0021-843X.111.4.637

49. Gros DF, Price M, Strachan M, Yuen EK, Milanak ME, Acierno R. Behavioral activation and therapeutic exposure: An investigation of relative symptom changes in PTSD and depression during the course of integrated behavioral activation, situational exposure, and imaginal exposure techniques. Behav Modif. 2012;36(4):580-99. [PMID:22679240] http://dx.doi.org/10.1177/0145445512448097

50. Barlow DH, Farchione TJ, Fairholme CP, Ellard KK, Boisseau CL, Allen LB, et al. Unified protocol for transdiagnostic treatment of emotional disorders: Therapist guide. New York (NY): Oxford University Press; 2010.

51. Mansell W, Harvey A, Watkins E, Shafran R. Conceptual foundations of the transdiagnostic approach to CBT. J Cogn Psychother. 2009;23(1):6-19.

http://dx.doi.org/10.1891/0889-8391.23.1.6

52. Gros DF. Development and initial evaluation of Transdiagnostic Behavior Therapy (TBT) for veterans with affective disorders. Psychiatry Res. 2014;220(1-2):275-82.

[PMID:25193379]

http://dx.doi.org/10.1016/j.psychres.2014.08.018

53. Gros DF, Milanak ME, Hershenberg R. Convergence of depression and anxiety symptoms in friendship dyads. J Soc Clin Psychol. 2013;32(10):1061-74. [PMID:25089072] http://dx.doi.org/10.1521/jscp.2013.32.10.1061

Submitted for publication May 21, 2014. Accepted in revised form April 23, 2015.

This article and any supplementary material should be cited as follows:

Raab PA, Mackintosh M, Gros DF, Morland LA. Impact of comorbid depression on quality of life in male combat Veterans with posttraumatic stress disorder. J Rehabil Res Dev. 2015;52(5):563-76.

http://dx.doi.org/10.1682/JRRD.2014.05.0130

ORCID: Phillip A. Raab, MA: 0000-0002-3722-4927; Margaret-Anne Mackintosh, PhD: 0000-0002-27253177; Daniel F. Gros, PhD: 0000-0003-3946-6716

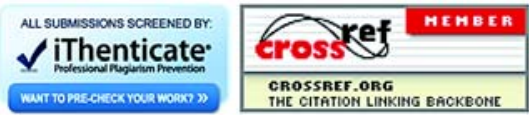

